\title{
Introduction to The World's Children Symposium
}

\author{
ROBERT HAGGERTY \\ William T. Grant Foundation, New York, New York 10022
}

For those who are skeptical as to whether we pediatricians in America should be engaged in the problems of the world or not, we have learned that every day nearly 40000 children throughout the world under the age of five die, largely of infectious diseases. We have begun to make some impact on this loss, but not nearly enough, through the Child Survival Program of UNICEF.

We in America should be concerned about the world's children for two primary reasons. First, wherever a child dies, it diminishes all of us, and we have the knowledge, skills, and resources that can be of help. Secondly, as the speakers today will point out, we also have a great deal to learn from engagement with the rest of the world. Many of the successes that have been demonstrated in other parts of the world could well be applied in our own country in areas where we still have much to achieve in child health.

American pediatricians have frequently worked on the world's stage. My mentor as well as Sam Katz's, Dr. Charles Janeway demonstrated this effectively in so many, many ways over his long career.

One point he made in his role in the International Pediatric Association was the nonpolitical nature of pediatrics. The International Pediatric Association, of which he was president, has always been open to pediatricians in all countries, irrespective of the type of government. There are obviously enormous difficulties in remaining politically neutral when so many of the problems of the world's children are tied to their socioeconomic condition. But he, like our speakers today, demonstrated that there is a middle way, a way of combining scientific knowledge as the basis for child help with the personal commitment to improve the lot of children all over the world.

Our first speaker, Dr. Donald A. Henderson, exemplifies this principal. He is the Dean of the School of Public Health at Johns Hopkins University. D. A. has made many contributions to world health, but is especially well-known for leading the fight that successfully resulted in the eradication of smallpox from the world. He continues to play a leading role in international health and will speak today on the Child Survival Revolution.

Whereas I certainly believe very much in the need to leave in place an infrastructure for services, I was also impressed, after hearing the stories of the Child Survival Program in different countries, that in many nations, the president of the country used the television to initiate the first mass immunization campaign and set the stage for continued implementation of the program by setting a good example.

Dr. Carl Gajdusek is a senior research fellow at NIH. He has the rare distinction of being one of the few pediatricians who have won the Nobel Prize, in his case, for discovering a whole new group of the slow viruses as a cause of human disease, Kuru. That is hardly the limit of his interest or what he is going to talk to us about today. He has also studied the way children learn in Stone Age societies. He is concerned about the world economy. In fact, there is very little he is not concerned and articulate about, and he is what I call a lateral thinker, going in directions rather different than most of us. He will be provocative, indeed perhaps outrageous, but he will always stimulate us with new ideas. He will speak on the paradoxes of aspirations for and of children in preliterate and isolated cultures. 